\title{
High uterosacral ligament hysteropexy for the management of pelvic organ prolapse
}

\author{
Naveen Kachro0 ${ }^{1,2}$, Samantha Raffee ${ }^{1}$, Solafa Elshatanoufy ${ }^{1}$, Humphrey Atiemo ${ }^{1}$ \\ ${ }^{1}$ Vattikuti Urology Institute, Henry Ford Hospital, Detroit, MI, USA; ${ }^{2}$ Glickman Urological and Kidney \\ Institute, Cleveland Clinic, Cleveland, OH, USA
}

\section{ABSTRACT}

Objective: To demonstrate our transvaginal high uterosacral ligament (HUL) hysteropexy technique as an alternative mesh-free uterine-preserving pelvic organ prolapse (POP) repair approach and present our institutional outcomes. Concurrent hysterectomy with POP repair is controversial as uterine-preserving techniques may beneficially allow fertility, body image and sexual function preservation $(1,2)$.

Materials and Methods: This video illustrates a step-by-step sequence of our HUL hysteropexy technique in a symptomatic Stage III POP patient. Retrospective single-institution, single-surgeon analysis of patients treated by either HUL hysteropexy or hysterectomy with HUL suspension for symptomatic prolapse was performed with minimum 2 years of follow-up. Patient demographics, operative characteristics, pre and post-operative POP-Q evaluation, American Urological Association Symptom scores (AUASS) and post-operative Pelvic Floor Distress Inventory (PFDI-20) were compared. Results: Surgery time was 3 hours 24 minutes. No immediate/early complications were noted, with successful repair on follow-up. Outcomes of 18 patients (10 HUL hysteropexy, 8 hysterectomy and HUL suspension) were assessed (Supplemental Table). The only baseline difference was a lower body mass index in the HUL hysteropexy cohort (25.8 vs. $35.8 \mathrm{~kg} /$ $\mathrm{m} 2, \mathrm{p}=0.008$ ). In the HUL hysteropexy cohort, blood loss (mean: $58 \mathrm{vs.} 205 \mathrm{ml}, \mathrm{p}=0.00086$ ) and operative time (190.4 vs. 279.1 minutes, $\mathrm{p}=0.0021$ ) were significantly reduced. There was no difference in post-operative AUASS, POP-Q or PFDI20 at 2 years.

Conclusion: We present our HUL hysteropexy technique. Although limited by sample size and retrospective design, resulted in significantly reduced blood loss and operative time with comparable post-operative 2 year outcomes to nonuterine-preserving techniques. In our opinion, HUL hysteropexy is a safe, durable POP management option for women without significant endometrial pathology risk factors.

\section{ACKNOWLEDGEMENTS}

Patient's consent: The patient gave full consent to all videos and images that have been submitted in advance of the video recording of the procedure.

Institutional Review Board Approval: Approval was obtained from the Henry Ford Hospital Institutional Review Board for this study - Study IRB\#9827

\section{CONFLICT OF INTEREST}

None declared. 


\section{REFERENCES}

1. Milani R, Frigerio M, Manodoro S, Cola A, Spelzini F. Transvaginal uterosacral ligament hysteropexy: a retrospective feasibility study. Int Urogynecol J. 2017; 28:73-6.
2. Costantini E, Porena M, Lazzeri M, Mearini L, Bini V, Zucchi A. Changes in female sexual function after pelvic organ prolapse repair: role of hysterectomy. Int Urogynecol J. 2013;24:1481-7.

\section{ARTICLE INFO}

iD Naveen Kachroo

http://orcid.org/0000-0002-9142-513X

Available at: http://www.intbrazjurol.com.br/video-section/20200384_Kachroo_et_al

Int Braz J Urol. 2021; 47 (Video \#14): 902-4

Submitted for publication:

May 07, 2020

Accepted after revision:

July 25,2020

Published as Ahead of Print:

March 10, 2021
Correspondence address: Humphrey Atiemo, MD Vattikuti Urology Institute, Henry Ford Hospital, 2799 W Grand Blvd Detroit, MI 48202, USA Fax: 313-916-4900 E-mail: hatiemo1@hfhs.org 


\section{SUPPLEMENTAL TABLE}

Table 1 - Comparison of outcomes of women undergoing high uterosacral ligament hysteropexy versus hysterectomy and high uterosacral ligament suspension for pelvic organ prolapse repair.

\begin{tabular}{|c|c|c|c|}
\hline & $\begin{array}{c}\text { High } \\
\text { Uterosacral Ligament } \\
\text { Hysteropexy }\end{array}$ & $\begin{array}{c}\text { High } \\
\text { Uterosacral Ligament Suspension with } \\
\text { Hysterectomy }\end{array}$ & p-value \\
\hline Number & 10 & 8 & \\
\hline Mean Age (years) ${ }^{\mathrm{a}}$ & $69.1 \pm 14.18$ & $65 \pm 13.00$ & 0.562 \\
\hline Mean BMI $\left(\mathrm{kg} / \mathrm{m}^{2}\right)^{\star a}$ & $25.81 \pm 4.48$ & $35.84 \pm 6.72$ & 0.008 \\
\hline Median Parity & 3 & 3 & \\
\hline \multirow[t]{3}{*}{ Race } & Caucasian: 11 & Caucasian: 6 & \\
\hline & African American: 3 & African American: 4 & \\
\hline & & Other: 3 & \\
\hline \multirow[t]{3}{*}{ Preop POP-Q Stage } & Stage $2: 30 \%$ & Stage $2: 25 \%$ & \\
\hline & Stage $3: 50 \%$ & Stage 3: $75 \%$ & 0.306 \\
\hline & Stage $4: 20 \%$ & Stage 4: 0 & \\
\hline AUASS Pre-opa & $19.9 \pm 6.06$ & $14.5 \pm 9.15$ & 0.230 \\
\hline AUASS Post-opa & $11.13 \pm 9.38$ & $13.00 \pm 6.24$ & 0.453 \\
\hline Operative Time (minutes) ${ }^{\star a}$ & $190.40 \pm 41.89$ & $279.13 \pm 39.01$ & 0.0021 \\
\hline $\mathrm{EBL}(\mathrm{mL})^{\star a}$ & $57.50 \pm 29.08$ & $205.00 \pm 94.38$ & 0.00086 \\
\hline Mean Follow-up (months) & 33.25 & 36.71 & 0.410 \\
\hline \multirow{4}{*}{$\begin{array}{l}\text { Post-op PopQ stage (at } 2 \text { years } \\
\text { in pts not requiring reoperation) }\end{array}$} & Stage 0: $0 \%$ & Stage 0: $0 \%$ & \multirow{4}{*}{0.282} \\
\hline & Stage 1: $12.5 \%$ & Stage 1: $50 \%$ & \\
\hline & Stage 2: $87.5 \%$ & Stage 2: $50 \%$ & \\
\hline & Stage $3+4: 0 \%$ & Stage $3+4: 0 \%$ & \\
\hline $\begin{array}{l}\text { Post-op PFDI-20 (at } 2 \text { years in } \\
\text { pts not requiring reoperation) }\end{array}$ & $18.50 \pm 17.61$ & $26.93 \pm 16.16$ & 0.483 \\
\hline
\end{tabular}

BMI = Body Mass Index; EBL = Estimated Blood Loss; AUASS = American Urological Association; $\mathbf{P O P Q}=$ Pelvic Organ Prolapse Quantification; PFDI = Pelvic Floor Distress Inventory.

${ }^{*}$ statistically significant difference

aMean +/- Standard Deviation 